\title{
HIGH-RESOLUTION MID-INFRARED IMAGING OF THE ASYMPTOTIC GIANT BRANCH STAR RV BOOTIS WITH THE STEWARD OBSERVATORY ADAPTIVE OPTICS SYSTEM
}

\author{
B. A. Biller, L. M. Close, A. Li, ${ }^{1}$ J. H. Bieging, W. F. Hoffmann, P. M. Hinz, D. Miller, \\ G. Brusa, M. Lloyd-Hart, F. Wildi, D. Potter, and B. D. Oppenheimer \\ Steward Observatory, University of Arizona, 933 North Cherry Avenue, Tucson, AZ 85721; bbiller@as.arizona.edu \\ Received 2003 August 28; accepted 2004 October 6
}

\begin{abstract}
We present high-resolution $\left(\sim 00^{\prime \prime} 1\right)$, very high Strehl ratio $(0.97 \pm 0.03)$ mid-IR adaptive optics $(A O)$ images of the asymptotic giant branch (AGB) star RV Boo utilizing the MMT adaptive secondary AO system. RV Boo was observed at a number of wavelengths over two epochs $(9.8 \mu \mathrm{m}$ in 2003 May and 8.8, 9.8, and $11.7 \mu \mathrm{m}$ in 2004 February) and appeared slightly extended at all wavelengths. While the extension is very slight at 8.8 and $11.7 \mu \mathrm{m}$, the extension is somewhat more pronounced at $9.8 \mu \mathrm{m}$. With such high Strehl ratios, we can achieve superresolutions of 0 ." 1 by deconvolving RV Boo with a point-spread function (PSF) derived from an unresolved star. We tentatively resolve RV Boo into a $0^{\prime \prime} 16 \mathrm{FWHM}$ extension at a position angle of $120^{\circ}$. At a distance of $390_{-100}^{+250} \mathrm{pc}$, this corresponds to a FWHM of $60_{-15}^{+40} \mathrm{AU}$. We measure a total flux at $9.8 \mu \mathrm{m}$ of $145 \pm 24 \mathrm{Jy}$ for the disk and star. Based on a dust thermal emission model for the observed IR spectral energy distribution and the $9.8 \mu \mathrm{m} \mathrm{AO}$ image, we derive a disk dust mass of $1.6 \times 10^{-6} M_{\odot}$ and an inclination of $30^{\circ}-45^{\circ}$ from edge-on. We discuss whether the dust disk observed around RV Boo is an example of the early stages in the formation of asymmetric structure in planetary nebulae.
\end{abstract}

Subject headings: infrared: stars — instrumentation: adaptive optics — techniques: high angular resolution

Online material: color figures

\section{INTRODUCTION}

Extensive mass loss in the asymptotic giant branch (AGB) phase has been well established. However, the mode (or modes) by which this mass loss occurs is less well known. While some objects present $\mathrm{CO}$ lines indicative of spherically symmetric mass loss, other objects display much more complicated massloss line profiles (Knapp et al. 1998; Kerschbaum \& Olofsson 1999; Olofsson et al. 2002). A small number of AGB and postAGB stars display a particular type of anomalous $\mathrm{CO}$ features: a very narrow peak (as narrow as $\sim 1 \mathrm{~km} \mathrm{~s}^{-1}$, see Kahane et al. [1998], but generally $\left.\leq 5 \mathrm{~km} \mathrm{~s}^{-1}\right)$, with or without a broader underlying pedestal feature (with widths of $10-20 \mathrm{~km} \mathrm{~s}^{-1}$ ). Stars that display just the narrow peak include AC Her, BM Gem, and the Red Rectangle. X Her (Kahane \& Jura 1996), RV Boo (Bergman et al. 2000), EP Aqr, RS Cnc, and IRC +50049 display narrow peaks as well as underlying broader components. Jura \& Kahane (1999) interpret the narrow features in these objects as reservoirs of dust and molecular gas that are nearly at rest with respect to the central AGB stars. They suggest that a binary companion is necessary in these cases in order to entrain gas and dust into a circumbinary disk (Morris 1987; Mastrodemos \& Morris 1998, 1999); both AC Her and the Red Rectangle have companions. The broader CO line components are interpreted as spherical outflows or in some cases (RS Cnc and $\mathrm{X}$ Her) as bipolar outflows (Kahane \& Jura 1996). RV Boo is unusual, since interferometric images of its $\mathrm{CO}$ emission suggest the presence of a large disk in Keplerian rotation (Bergman et al. 2000). Interestingly, another sort of asymmetry in AGB star envelopes has recently been imaged: a small bipolar outflow

\footnotetext{
${ }^{1}$ Theoretical Astrophysics Program, Steward Observatory and Lunar and Planetary Laboratory, University of Arizona, Tucson, AZ 85721; agli@lp1. arizona.edu.
}

observed in the near-IR around the AGB star IRC +10011 (Hofmann et al. 2001; Vinkovic et al. 2004).

Determining the spatial structure of winds around AGB stars is important for constraining models of bipolar planetary nebulae (PNs). Generalized interacting stellar winds (GISW) models of PNs invoke some initial structure that can collimate and shape the fast winds produced by these objects. In these models, the existence or nonexistence of this structure regulates whether the forming PN acquires a round, elliptical, or bipolar morphology (Balick \& Frank 2002). This preexisting structure is likely to have formed by the end of the central object's AGB stage, since a sizable percentage of proto-PNs display bipolar reflection nebulae. Could the disks and molecular reservoirs observed in $\mathrm{CO}$ around AGB stars be the initial stages in the formation of such preexisting structure? The molecular reservoirs observed by Kahane et al. (1998) may be the diffuse precursors to the formation of a denser disk or torus around the star at the end of the AGB phase. Such dense disks might be capable of collimating the fast winds produced when these objects evolve to the PN phase.

At mid-IR wavelengths, we expect dusty disks around AGB and post-AGB stars to be detected in thermal emission. Do we observe the disks implied by $\mathrm{CO}$ observations at these wavelengths? Meixner et al. (1999) observed a number of proto-PNs in the mid-IR. In those that were resolved, they found two primary mid-IR morphologies: core/elliptical and toroidal. Ueta et al. (2000) found that each of these mid-IR morphologies also corresponds to a specific optical morphology. The optically thick core/elliptical mid-IR morphologies possessed bipolar reflection nebulae and heavily obscured central stars. The optically thin toroidal mid-IR morphologies possessed elliptical reflection nebulae and nonobscured central stars. In a number of cases dusty disks have not been observed directly but may collimate reflection nebulae; for instance, Roddier et al. (1995) 

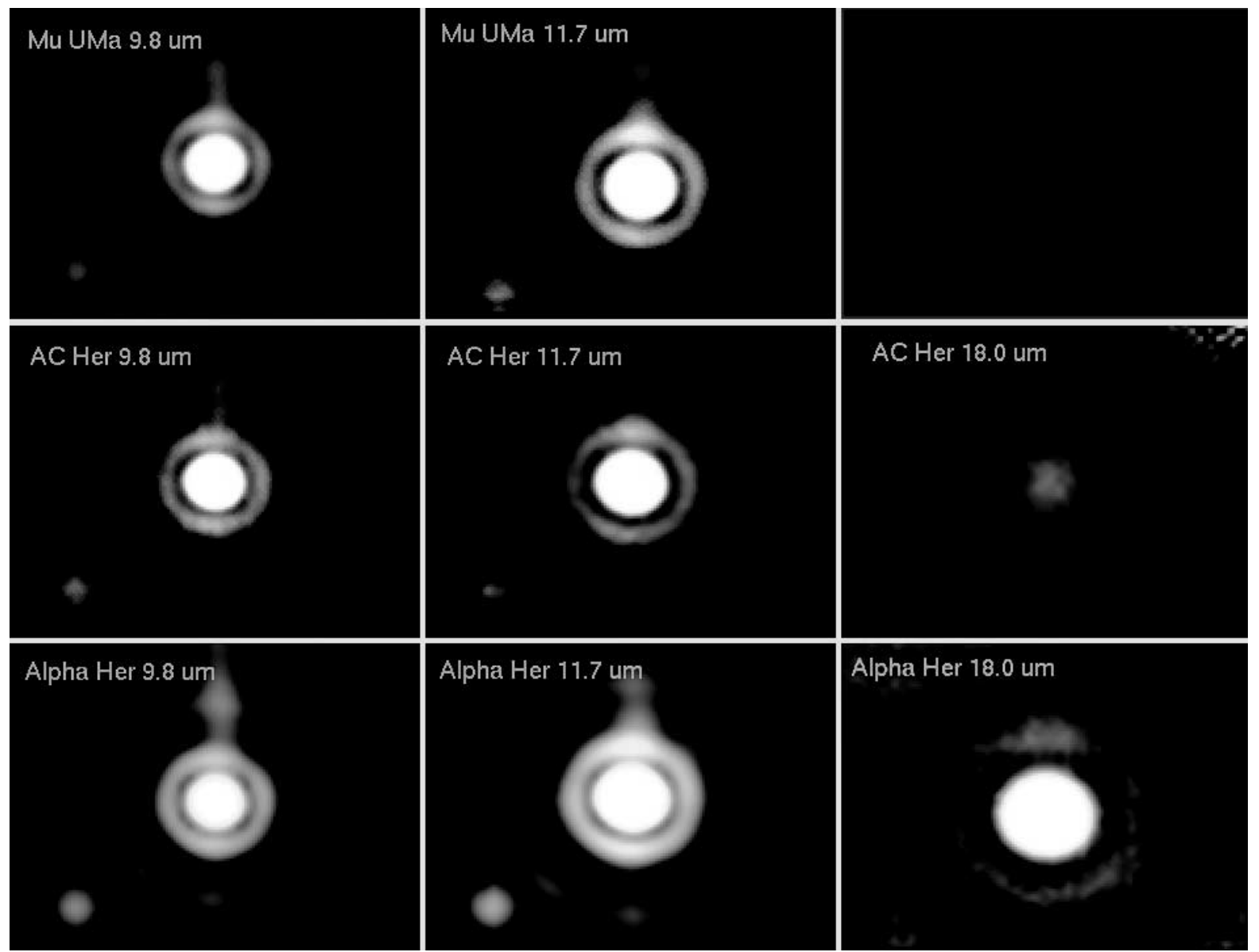

FIG. 1. - The 9.8, 11.7, and $18 \mu \mathrm{m}$ images of the PSF stars $\mu$ UMa, AC Her, and $\alpha$ Her as observed at the MMT. The box size of the MMT images is 1".5 $\times 11^{\prime \prime} 0$. The faint point source in the lower left of each MMT image is a MIRAC3 ghost (Fig. 1 of Close et al. 2003). [See the electronic edition of the Journal for a color version of this figure.]

observe double-lobed reflection in the near-IR ( $J$ and $K$ bands) around the Red Rectangle (IRAS 06176-1036) and Frosty Leo (IRAS 09371+1212). Meixner et al. (1999) resolved the Red Rectangle into an unresolved core and an extended elliptical nebulosity but found Frosty Leo to be unresolved.

$\mathrm{RV}$ Boo is an O-rich type b semiregular (SRb) variable. It varies in luminosity and in spectral class between M5 III and M7 III with a period of $~ 140$ days. Previously, Bergman et al. (2000) observed a 4" diameter disk in CO around RV Boo. They interpret this disk as possibly the first known Keplerian disk around an AGB star. The resolution in $\mathrm{CO}$ radio lines is limited to $\sim 1^{\prime \prime}-2^{\prime \prime}$, even using interferometry; by observing at high resolution in the mid-IR, we can probe the structure of this disk on much finer scales.

Using the unique adaptive secondary mirror adaptive optics (AO) system at the $6.5 \mathrm{~m}$ MMT (Wildi et al. 2003; Brusa et al. 2003), we can observe AGB stars at mid-IR wavelengths with $\sim 0$.'1 resolution (Close et al. 2003). Through deconvolution, the nearly perfect images (Strehl ratio $\sim 0.97 \pm 0.03$ ) produced with $\mathrm{AO}$ at the MMT allow resolutions better than that of the FWHM of the diffraction limit of the telescope. With such resolutions, we can probe AGB star and proto-PN morphologies on finer scales than ever before possible in the mid-IR. Here we present the first AO high-resolution images of RV Boo.

\section{OBSERVATIONS AND DATA REDUCTION}

Data were taken on the night of 2003 May 13 (UT) at the $6.5 \mathrm{~m}$ MMT using the adaptive secondary mirror AO system with the BLINC-MIRAC3 camera (Hoffman et al. 1998; Hinz et al. 2000). The adaptive secondary corrected the first 52 system modes at $550 \mathrm{~Hz}$ and achieved Strehl ratios as high as $0.97 \pm 0.03$ from 8.8 to $18 \mu \mathrm{m}$. These Strehl ratios are the highest ever presented in the literature; previous Strehl ratios for large telescopes have rarely exceeded 0.7 at any wavelength. Our unique ability to do AO correction at $10 \mu \mathrm{m}$ leads to very high Strehl ratios regardless of the seeing, air mass, or wind (Close et al. 2003). With the very stable point-spread functions (PSFs) that result from such high Strehl ratios, it is possible to detect structures with spatial scales smaller than the diffraction-limited FWHM $(\sim 0.98 \lambda / D$ rad $)$ through the use of deconvolution.

Images of all the PSF stars observed on 2003 May 13 are displayed in Figure 1. To further illustrate stability, we subtracted one PSF star ( $\alpha$ Her) from another observed later in the evening (AC Her). The residuals are displayed in Figure 2. The residual flux after PSF subtraction is $<0.5 \%$ of AC Her's original flux. Similar residuals resulted from PSF subtractions at 9.8 and $18 \mu \mathrm{m}$. Based on these excellent subtractions, we conclude that the PSF obtained from the MIRAC3 camera with the MMT adaptive secondary AO system is extremely stable.

RV Boo was observed in the $9.8 \mu \mathrm{m}$ wavelength band. Point sources $\mu \mathrm{UMa}$ and $\alpha$ Her were observed in this band before and after RV Boo to use as PSF calibrators. $\alpha$ Her is a relatively wide binary with a separation of 4".7 (Jeffers \& Vasilevskis 1978); the brighter component was used as a PSF, while the fainter component falls outside our field of view. We note that $\alpha$ Her also does not possess a dust shell or other extended 


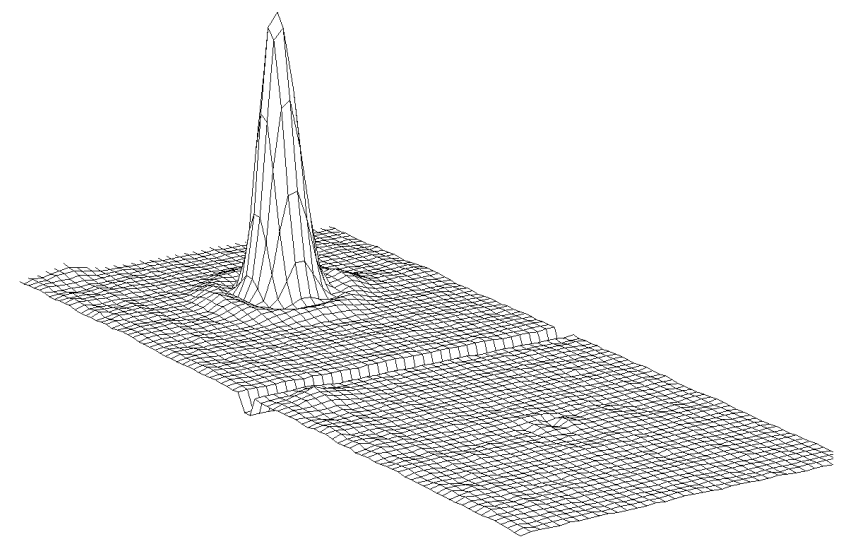

FIG. 2.-The $11.7 \mu \mathrm{m}$ PSF of AC Her before (left) and after (right) PSF subtraction (using $\alpha$ Her as the PSF) with DAOPHOT's ALLSTAR task. The residual flux after PSF subtraction is $<0.5 \%$ of AC Her's original flux. Similar residuals resulted from PSF subtractions at 9.8 and $18 \mu \mathrm{m}$. Based on these excellent subtractions, we conclude that the PSF obtained from the MIRAC3 camera with the MMT adaptive secondary AO system is extremely stable. Note that the small ghost image to the lower left in each frame is not subtracted to show that the vertical scales are the same for both images (Fig. 3 of Close et al. 2003).

structure (Close et al. 2003). To eliminate the high sky background at mid-IR wavelengths, we used a standard chopping/nodding scheme and flat-fielded our data. A chopping frequency of $1 \mathrm{~Hz}$ (throw $\sim 20^{\prime \prime}$ ) was used with a nodding cycle of $60 \mathrm{~s}$ (throw $\left.\sim 6^{\prime \prime}-8^{\prime \prime}\right)$. To avoid saturation of the high sky background, a base integration time of $29 \mathrm{~ms}$ was used. These images were coadded to produce an output frame every $15 \mathrm{~s}$. For RV Boo, four $15 \mathrm{~s}$ integrations were taken at each of 8 nod positions, giving a total exposure time of 8 minutes. $\mu$ UMa was observed using 8 nod positions for a total exposure time of 8 minutes. $\alpha$ Her was observed using 4 nod positions for a total exposure time of 4 minutes. We used the internal BLINC cold chopper and kept the AO in closed loop for both chop and nod beam positions.

Flat fields were taken the night of 2003 May 15 (UT). The base integration time was set to $10 \mathrm{~ms}$. These images were coadded every $2 \mathrm{~s}$. Flats were taken of the inside of the dome (hot) and the sky (cold). The sky flats were subtracted from the dome flats. The resulting flat fields were normalized by the mean.

To determine an astrometric calibration, we used our 2002 November 25 (UT) observations of the binary star WDS $02589+$ 2137 BU. These data were taken in the $M$ band using MIRAC with the MMT adaptive secondary AO system. At the time of observation, the binary had a position angle of $269^{\circ}$ and a separation of 0.509 (Mason et al. 2001). To align our RV Boo data with north, we must rotate it by $270^{\circ}-$ the parallactic angle at the time of observation. We also determine a plate scale of 88 mas pixel $^{-1}$ from this standard.

Data were reduced using a custom IRAF pipeline that first flat-fields and removes bad pixels. After the pipeline completes these basic data reduction tasks, it then rotates the nod images by $270^{\circ}$ - the parallactic angle and co-adds them so that north is up and east is left. A co-added image of RV Boo alongside similar images of the PSF stars $\mu$ Uma and $\alpha$ Her as well as the AGB star AC Her (Close et al. 2003) is presented in Figure 3. The vertical axis is telescope altitude, while the horizontal axis is telescope azimuth. RV Boo appears slightly extended (FWHM $\sim 4$ pixels) relative to the PSF stars (FWHM $\sim 3.8$ pixels). All three PSF stars are very slightly elliptical (eccentricity $\sim 2 \%$ ) along the horizontal (azimuthal) direction; this is a systematic instrumental feature of the PSF. However, RV Boo appears some-

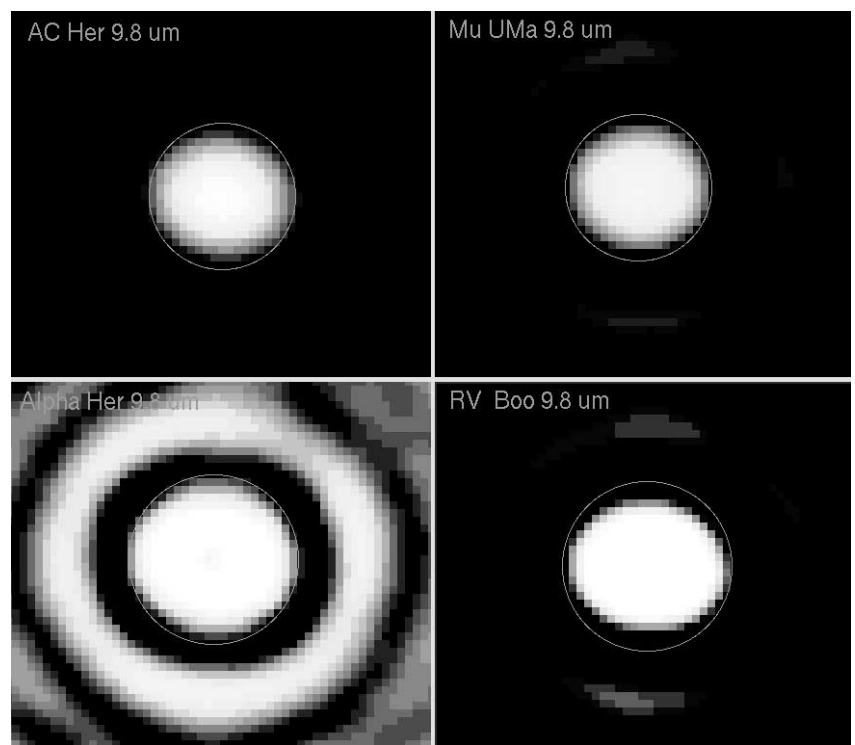

FIG. 3.-AO images of RV Boo, $\mu$ UMa, $\alpha$ Her, and AC Her at $9.8 \mu \mathrm{m}$. The vertical axis is telescope altitude, while the horizontal axis is telescope azimuth. The images are Fourier filtered; an image smoothed with a Gaussian with a 3 pixel FWHM was subtracted from each of the original images in order to remove variations on large spatial wavelengths. All images are shown on a logarithmic scale; the bright ring around the images is the first Airy ring. Note that RV Boo appears nominally extended relative to the other stars. The PSF star $\alpha$ Her is slightly saturated. A 0.7 diameter circle is overlaid on each image to help aid the eye. [See the electronic edition of the Journal for a color version of this figure.]

what more elliptical (eccentricity 5\%) than the other three stars. We argue that this extension and slight ellipticity is indicative of actual physical structure. The observed extension lies near the limit of resolution of the telescope, however, and is broadened by diffraction. To discern the actual small-scale structure of the extension around RV Boo, we must deconvolve it with a PSF star.

In order to determine whether the extension we see is real (and not just the result of a vibration in the telescope mount, for instance), we deconvolved each of the $8 \mathrm{RV}$ Boo nod images with the $\mu$ UMa PSF. After subpixel interpolation by a factor of 3 (to a new plate scale of 29.3 mas pixel $^{-1}$ ), we used the Lucy deconvolution algorithm in IRAF with 1000 iterations. We chose to deconvolve for 1000 iterations for two reasons. First, object properties (position angle and deconvolution) vary rapidly up until the $\sim 800$ th iteration. By the 1000th iteration, properties have converged. Second, we convolved a set of thermal disk models at inclination angles from edge-on of $5^{\circ}$, $15^{\circ}$, and $30^{\circ}$ (see $\S 4$ for details on our modeling) with the $\mu$ UMa PSF, then deconvolved for 100, 500, 1000, 1500, and 2000 iterations using AC Her (Close et al. 2003) as the PSF. The 1000 iteration deconvolutions best re-created the position angles and eccentricities of our models.

If the extension is real, we expect the position angle of the deconvolved semimajor axis to track the parallactic angle as the sky rotates between exposures. We measured position angle and eccentricity for each deconvolution of the eight individual RV Boo nod images using the imexam tool in IRAF. The imexam tool is only accurate for measuring isophote semimajor position angles to within $\pm 6^{\circ}$ of accuracy. We calculate $\sigma=5^{\prime \prime}$. 65 as our error in position-angle measurements. This error was determined by stretching an image of $\mu$ UMa to $5 \%$ eccentricity (about the eccentricity of RV Boo previous to deconvolution), rotating it through a number of angles, and then taking the 


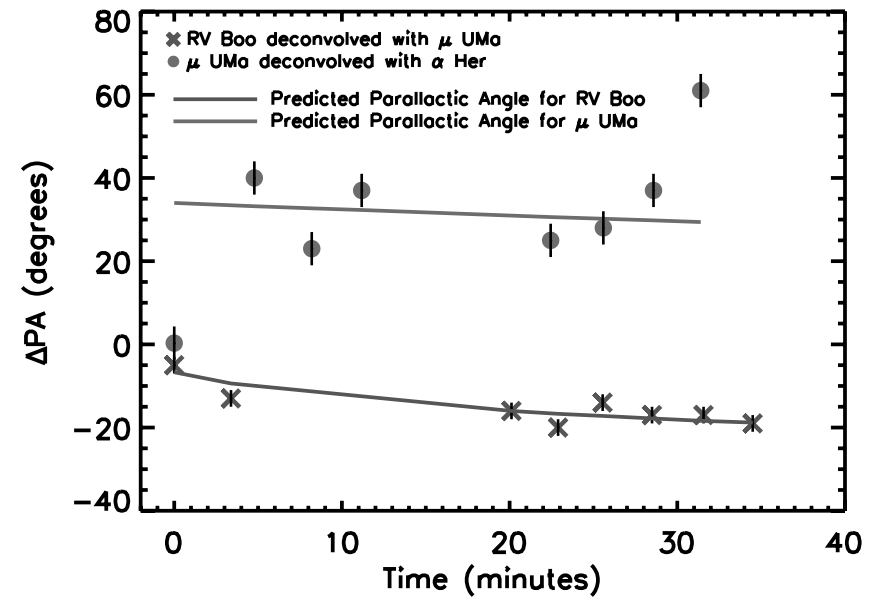

FIG. 4.-Position angle of the semimajor axis vs. time (after first observation) for deconvolved RV Boo and $\mu$ UMa nod images. Measured position angles are represented as crosses for RV Boo and circles for $\mu \mathrm{UMa}$; solid lines depict the predicted parallactic angle as a function of time during the observations. Note that the $\triangle$ PAs measured for RV Boo track the parallactic angle much more closely than those measured for $\mu \mathrm{UMa}$, with a reduced $\chi^{2}$ value of 0.207 for RV Boo deconvolved with $\mu$ UMa vs. a reduced $\chi^{2}$ value of 13.2 for $\mu$ UMa deconvolved with $\alpha$ Her. This implies that the elongation observed was really associated with RV Boo (since it was rotating along with the sky) and is not a PSF artifact. [See the electronic edition of the Journal for a color version of this figure.]

standard deviation of the measured angle minus actual angle as the error.

We plot the behavior of the major axis position angle of the deconvolved image (hereafter $\triangle \mathrm{PA}$ ) and parallactic angle with time in Figure 4. We fit the expected parallactic angles (calculated using the Skycalc software package; Thorstensen 2001) to the position angle data for each star with a minimized $\chi^{2}$. Thus, $\triangle \mathrm{PA}$ measures how much the trend in position angle deviates from the trend in parallactic angle. Expected parallactic angles are plotted as solid lines; observed values of $\Delta \mathrm{PA}$ are plotted using a variety of points. We plot the behavior of $\triangle \mathrm{PA}$ for RV Boo deconvolved with $\mu \mathrm{UMa}$ as the PSF. As a comparison, we plot $\Delta \mathrm{PA}$ for $\mu$ UMa deconvolved with $\alpha$ Her, a PSF deconvolving a PSF (both apparent point sources). The $\triangle \mathrm{PA}$ values measured from the RV Boo deconvolutions follow the correct rotation of the sky with a reduced $\chi^{2}$ of 0.207 for the deconvolution with $\mu$ UMa. Thus, RV Boo's extension is rotating on the sky to within a probability of $98 \%$. In contrast, the $\triangle \mathrm{PA}$ values measured from the PSF and PSF deconvolution show much more scatter and have a best fit to the parallactic angles with a reduced $\chi^{2}$ of 13.2. Hence, the extension of a PSF deconvolved with a PSF is purely an artifact; the probability that $\triangle \mathrm{PA}$ for $\mu \mathrm{UMa}$ deconvolved with $\alpha$ Her rotates on the sky is less than $0.1 \%$ as one would expect.

The variation of PSF FWHM with source eccentricity for $\mathrm{RV}$ Boo, $\mu \mathrm{UMa}, \alpha$ Her, and AC Her is presented in Figure 5. These quantities are also presented in Figure 5 for the best-fit thermal emission model of the RV Boo disk convolved with the $\mu$ UMa PSF (see $\S 4$ ). An extended source should have a larger FWHM and possibly a higher eccentricity (depending on position angle, source shape, and source inclination angle) than a point source; RV Boo indeed shows this trend. The RV Boo disk models accurately re-create the FWHM and eccentricity of the RV Boo data. Models were fit to the deconvolved data; the match between convolved model and undeconvolved data implies that 1000 iterations of the Lucy algorithm produces an accurate de-

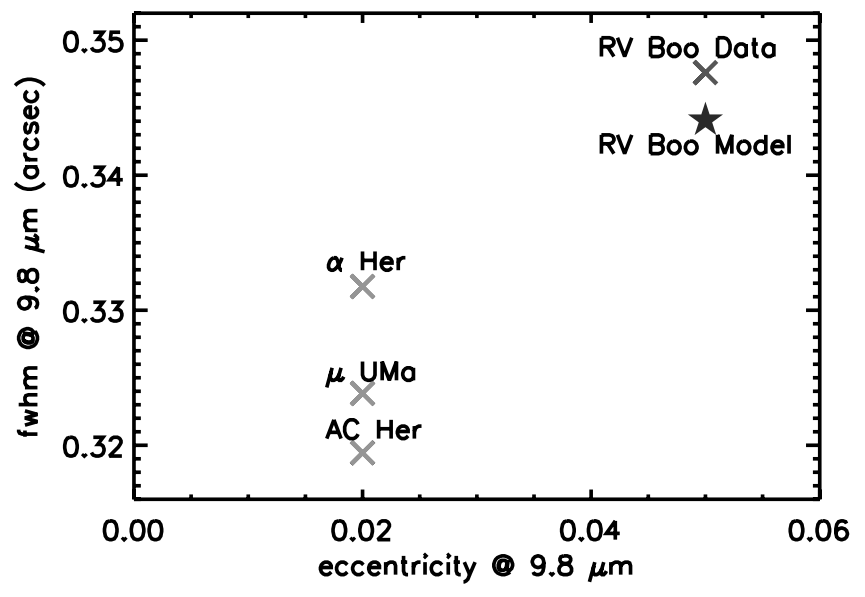

FIG. 5.-Eccentricity vs. PSF FWHM for RV Boo, $\mu$ UMa, $\alpha$ Her, and AC Her images. The best-fit thermal emission model of RV Boo convolved with the $\mu$ UMa PSF is also plotted (see $\S 4$ for details on modeling). The three PSF stars are plotted as crosses. The RV Boo data is plotted as a cross, and the RV Boo model is plotted as a star. FWHM is measured by a Gaussian fit to the enclosed flux at each radius. RV Boo appears slightly extended and has a significantly higher eccentricity and FWHM than the other stars. [See the electronic edition of the Journal for a color version of this figure.]

convolution of the RV Boo data. On the basis of the rotation of the extension with the sky and its non-PSF FWHM and eccentricity, we tentatively conclude that the extension we observe around RV Boo is indeed real and not an artifact of the telescope. However, to strengthen this conclusion, we reobserved RV Boo at a variety of wavelengths at another epoch.

\section{FOLLOW-UP OBSERVATIONS}

RV Boo was reobserved on the night of 2004 February 2 (UT) at the $6.5 \mathrm{~m}$ MMT using the adaptive secondary mirror AO system with the BLINC-MIRAC3 camera (Hoffman et al. 1998; Hinz et al. 2000). Strehl ratios were similar to those observed during the first observations; however, the MIRAC camera was used with a plate scale of 0 ". 079 (as opposed to $00^{\prime \prime} 088$ during the previous run). These data was taken during an engineering test and are of considerably lower quality (engineering grade rather than science grade) than the 2003 May data set.

As before, we used a standard chopping/nodding scheme. A chopping frequency of $1 \mathrm{~Hz}$ (throw $\sim 20^{\prime \prime}$ ) was used. Each object was observed at three different wavelengths $(8.8,9.8$, and $11.7 \mu \mathrm{m}$ ) and two different nod positions (throw $\sim 4^{\prime \prime}-5^{\prime \prime}$ ). To avoid saturation of the high sky background, a base integration time of 30-70 ms was used. These images were co-added to produce an output frame every $10 \mathrm{~s}$. For RV Boo, $10 \times 10 \mathrm{~s}$ integrations were taken at two nod positions for each wavelength, giving a total exposure time of $200 \mathrm{~s}$ per wavelength. $\alpha$ Boo was observed at two nod positions per wavelength for a total exposure time of $200 \mathrm{~s}$ per wavelength. We used the internal BLINC cold chopper and kept the AO in closed loop for all chop and nod beam positions. The data were reduced with the same custom IRAF pipeline as before. With shorter total integration times (200 s per wavelength for RV Boo during reobservations vs. $480 \mathrm{~s}$ at $9.8 \mu \mathrm{m}$ during the initial observations), our signal-to-noise ratio during the follow-up observations is only $\sim 65 \%$ that achieved during the initial observations.

PSF and data FWHMs for both the 2003 May and 2004 February data sets are presented in Table 1. We estimate an uncertainty in our measurements of \pm 6 mas from the scatter 
TABLE 1

FWHMS FOR RV BOO AND PSF STARS

\begin{tabular}{|c|c|c|c|c|c|c|}
\hline \multirow[b]{2}{*}{$\begin{array}{c}\lambda \\
(\mu \mathrm{m})\end{array}$} & \multicolumn{4}{|c|}{2003 MAY } & \multicolumn{2}{|c|}{2004 FEBRUARY } \\
\hline & $\begin{array}{c}\mu \mathrm{UMa} \\
\text { (PSF) }\end{array}$ & $\begin{array}{l}\alpha \text { Her } \\
\text { (PSF) }\end{array}$ & $\begin{array}{c}\text { AC Her } \\
\text { (PSF) }\end{array}$ & RV Boo & $\begin{array}{c}\alpha \text { Boo } \\
\text { (PSF) }\end{array}$ & RV Boo \\
\hline $8.8 \ldots \ldots \ldots \ldots$ & $\ldots$ & . & $\ldots$ & $\ldots$ & 0.292 & 0.297 \\
\hline $9.8 \ldots \ldots \ldots \ldots$ & 0.324 & 0.332 & 0.319 & 0.348 & 0.322 & 0.338 \\
\hline $11.7 \ldots \ldots \ldots$ & $\ldots$ & $\ldots$ & $\ldots$ & $\ldots$ & 0.382 & 0.393 \\
\hline
\end{tabular}

NoTE.-The uncertainty for all of the FWHMs is \pm 6 mas.

of the PSF FWHMs in the May data. The FWHM of RV Boo was slightly larger than that of the PSF in all wavelengths in February; however, the extension is within the measurement error for the 8.8 and $11.7 \mu \mathrm{m}$ data. The February RV Boo $9.8 \mu \mathrm{m}$ data possesses a FWHM comparable to that of the May RV Boo data; thus, we reobserve the slight extension found in May in our February data set. Perhaps it is not surprising that the object appears most extended at $9.8 \mu \mathrm{m}$, right at the center of a prominent silicon emission feature seen in the Infrared Space Observatory (ISO) spectrum (see $\S 4$ and Fig. 7). For RV Boo, we measured eccentricities of $\sim 2 \%$ in February versus $\sim 5 \%$ in May. This may have simply been a function of position angle during the observations. Unfortunately, the engineering grade observations of the only PSF $\operatorname{star}(\alpha$ Boo) were found to have low spatial frequency noise and were unsuitable to be used as a PSF for deconvolution. As a test, we deconvolved these observations using the $\mu$ UMa PSF from the previous observations. The February data possessed similar deconvolved source properties (FWHM, position angle, and eccentricity) compared to the May data.

\section{ANALYSIS}

The deconvolved image of RV Boo is presented in Figure 6. This image was produced by co-adding all of the RV Boo nod images after they had been deconvolved for 1000 iterations using the Lucy algorithm with $\mu \mathrm{UMa}$ as the PSF. (Because of the slight saturation of the $\alpha$ Her image, $\mu$ UMa was chosen as the PSF over $\alpha$ Her for this analysis.) After deconvolution, the extended structure appears to be a disk seen at an inclination angle between $30^{\circ}$ and $45^{\circ}$ from edge-on (inclination estimated from modeling; see below). From their $\mathrm{CO} J=2 \rightarrow 1$ interferometric data, Bergman et al. (2000) found evidence of a rotating disk around RV Boo in the form of a $\sim 4^{\prime \prime}$ diameter disk with a position angle of $\sim 150^{\circ}$. Using the imexam tool in IRAF, we measured a FWHM at $\lambda=9.8 \mu \mathrm{m}$ of 0.16 along the major axis and a position angle of $120^{\circ}$. The $9.8 \mu \mathrm{m}$ emission traces dust thermal reemission; RV Boo heats only a fraction of the dust in the entire $\mathrm{CO}$ disk to temperatures at which dust is luminous in the mid-IR. If circumstellar gas and dust are well mixed around RV Boo, we would expect to find a much smaller disk extent at $9.8 \mu \mathrm{m}$ than in $\mathrm{CO}$, since all of the $\mathrm{CO}$ will emit, but only the dust near enough to the star to be heated to temperatures of $\sim 300 \mathrm{~K}$ will emit at $9.8 \mu \mathrm{m}$. To map out more extension in the dust disk, observations at longer wavelengths (which trace a larger region of dust reemission) are necessary. We measure a total flux for RV Boo at $9.8 \mu \mathrm{m}$ of $145 \pm 24 \mathrm{Jy}$.

At a distance of $390_{-100}^{+250}$ pc (Perryman et al. 1997), the midIR disk of RV Boo subtends a FWHM of $60_{-15}^{+40} \mathrm{AU}$. RV Boo is a relatively nearby AGB star; it would be more difficult to observe a similar disk around a more distant star like AC Her, which is $\sim 2$ times more distant. Since RV Boo is so nearby relative to
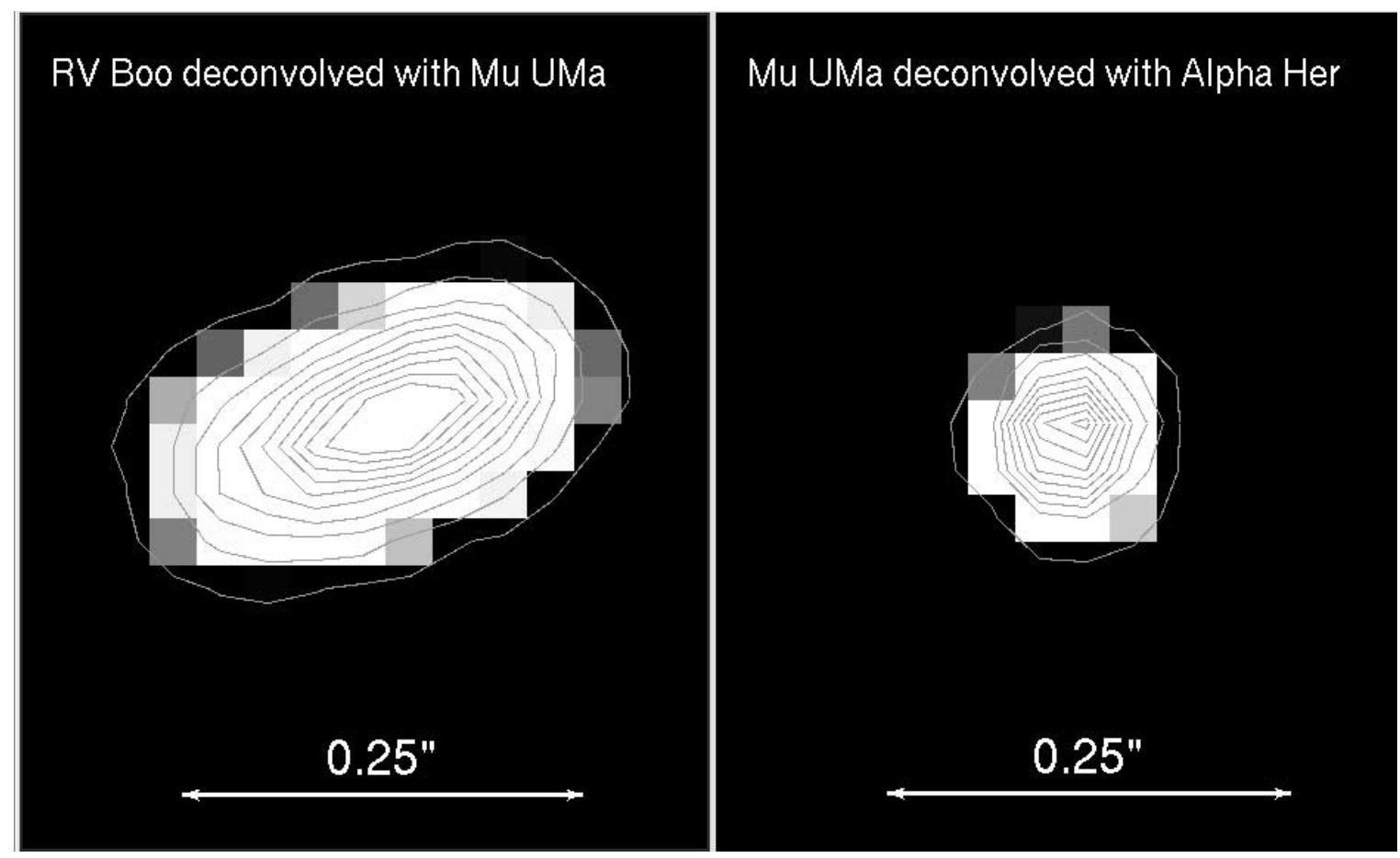

Fig. 6.-Deconvolved image of RV Boo. The plate scale (after magnification) is 29.3 mas pixel $^{-1}$. North is up; east is left. The disk is at a position angle of $120^{\circ}$ and has a major axis FWHM of $0^{\prime \prime} 16(\sim 60$ AU at $390 \mathrm{pc})$. A deconvolved image of $\mu$ UMa (deconvolved using $\alpha$ Her as a PSF) is shown on the right for comparison. For each object, there are 10 contours spaced linearly. For RV Boo, the lowest contour level is placed at $3 \%$ of the peak flux ( $275 \mathrm{Jy}$ arcsec ${ }^{-2}$ ) and the highest contour level is placed at $98 \%$ of the peak flux $\left(9640 \mathrm{Jy} \mathrm{arcsec}^{-2}\right)$. For $\mu \mathrm{UMa}$, the lowest contour level is placed at $1 \%$ of the peak flux ( $279 \mathrm{Jy}$ arcsec ${ }^{-2}$ ) and the highest contour level is placed at $95 \%$ of the peak flux $\left(2.24 \times 10^{4} \mathrm{Jy}_{\operatorname{arcsec}^{-2}}\right)$. After deconvolution, RV Boo appears extended, while the PSF still appears pointlike. [See the electronic edition of the Journal for a color version of this figure.] 


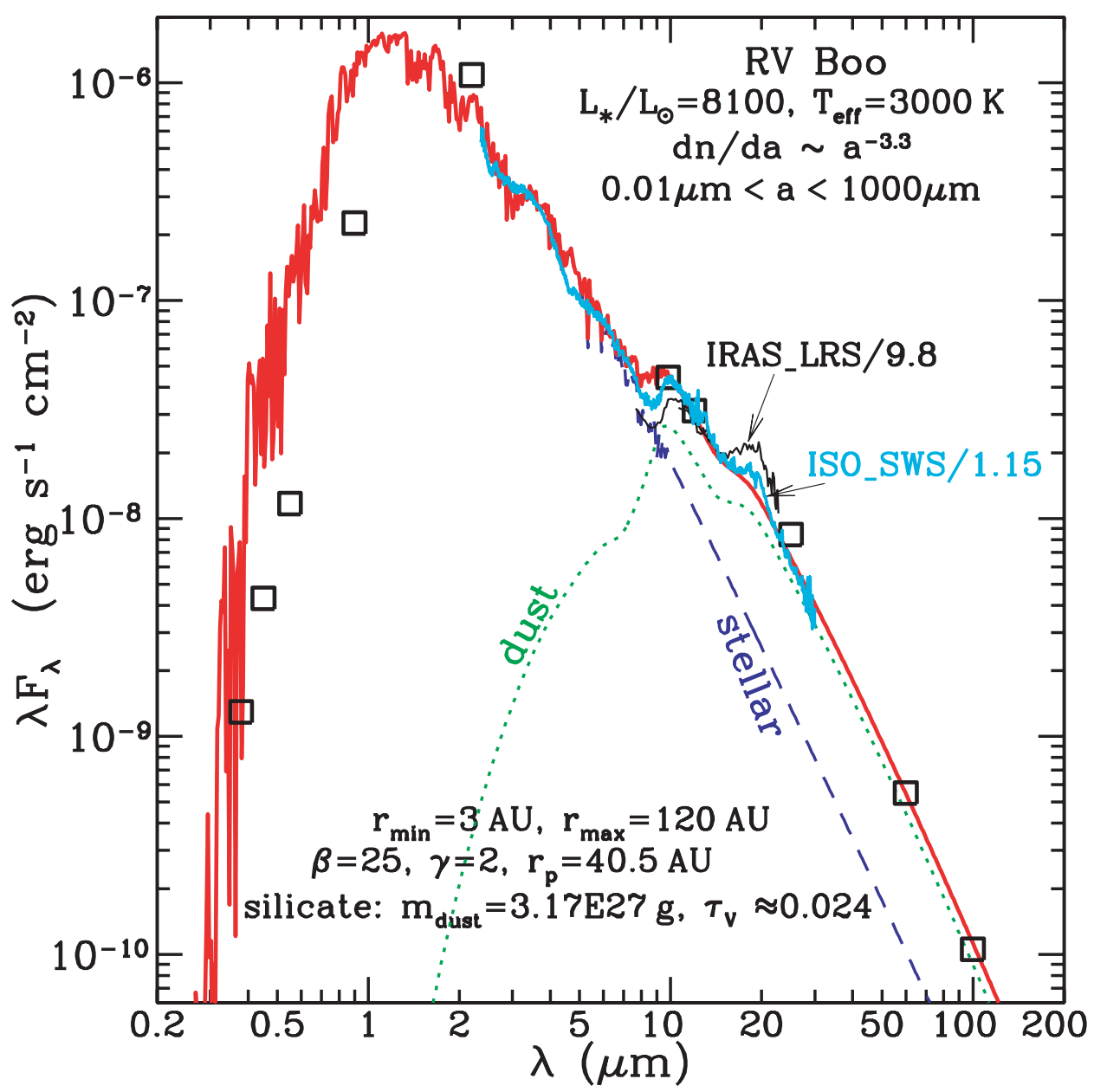

FIG. 7.- IR emission and model fit to the RV Boo disk. The open black squares are the $U, B, V, I$, and $K$ photometry (from the literature), our $9.8 \mu \mathrm{m}$ AO photometry, and the 12,25,60, and $100 \mu \mathrm{m}$ IRAS photometry. The thin solid black line is the IRAS LRS spectrum (reduced by a factor of 9.8 in order to agree with the IRAS $12 \mu \mathrm{m}$ data; see $\S 4$ ). The thin solid cyan line is the ISO SWS spectrum (reduced by a factor of 1.15 ; see $\S 4$; the spectrum longward of $30 \mu \mathrm{m}$ is too noisy and therefore not shown in this figure). The green dotted line is the dust model emission. The blue dashed line is the stellar photospheric spectrum approximated by the Kurucz model of $T_{\text {eff }}=3000 \mathrm{~K}$. The red solid line is the sum of the dust and stellar emission. At a distance of $d \approx 390 \mathrm{pc}$, this best-fit model has an inner edge at $3 \mathrm{AU}$ and a peak dust density at $r_{p} \approx 40.5 \mathrm{AU}$.

the distance of the average AGB star, it is not surprising that such small-scale structure usually is unresolved in other systems. Small mid-IR disks around AGB stars could be common but difficult to resolve.

We model the IR emission of RV Boo as an optically thin disk passively heated by the star. ${ }^{2}$ We approximate the stellar photosphere by the Kurucz (1979) model spectrum for M6IIIe stars with an effective temperature of $T_{\text {eff }}=3000 \mathrm{~K}$. The dust is taken to be amorphous silicate, since RV Boo is an M star. We assume a power-law dust size distribution $d n(a) / d a \propto a^{-\alpha}$ that is characterized by a lower cutoff $a_{\min }$, upper cutoff $a_{\max }$, and power-law index $\alpha .^{3}$ We take $a_{\min }=0.01 \mu \mathrm{m}$, since grains smaller than this will undergo single-photon heating (Draine \& Li 2001), while the observed IR spectral energy distribution of RV Boo does not appear to show evidence for stochastically

\footnotetext{
${ }^{2} \mathrm{RV}$ Boo has a $B-V$ color of $\sim 1.47 \mathrm{mag}$; this low reddening implies that the mid-IR-emitting region is not obscuring the entire star (as it would be if the dust was spherically distributed around the star) and is largely concentrated along one plane. Thus, we have explicitly modeled the IR emission around RV Boo as a disk. Since we have a priori considered a disk morphology, our model cannot constrain the vertical geometry of the emission.

${ }^{3}$ We assume all grains are spherical in shape with $a$ being their spherical radius.
}

heated dust, and $a_{\max }=1000 \mu \mathrm{m}$, since larger grains are not well constrained by the currently available IR photometry.

The dust spatial distribution is taken to be a modified power law $d n / d r \propto\left(1-r_{\min } / r\right)^{\beta}\left(r_{\min } / r\right)^{\gamma}$, where $r_{\min }$ is the inner boundary of the disk, which we take to be the location where silicate dust sublimates. For RV Boo with a luminosity $L_{*} \approx$ $8100 L_{\odot}$ (Bergman et al. 2000), submicron-sized silicate dust achieves an equilibrium temperature of $T \approx 1500 \mathrm{~K}$ and starts to sublimate at $r \approx 3 \mathrm{AU}$. Therefore, we take $r_{\min }=3 \mathrm{AU}$. This functional form has the advantage that on one hand it behaves like a power law $d n / d r \propto r^{-\gamma}$ at larger distances $(r \gg$ $\left.r_{\min }\right)$, and on the other hand it peaks at $r_{p}=r_{\min }(\beta+\gamma) / \gamma$, unlike the simple power law, which peaks at $r_{\min }$. The latter is unphysical, since one should not expect dust to pile up at $r_{\min }$ where dust sublimates. We take $\gamma=2$, as expected from a stationary outflow. We take $r_{\max }=120 \mathrm{AU}$, which is large enough for our dust IR emission modeling purpose, since there is very little dust beyond this outer boundary. Therefore, we have only two free parameters: $\alpha$, the power-law exponent for the dust size distribution, and $\beta$, the dust spatial distribution parameter that determines where the dust peaks.

Using the dielectric functions of Draine \& Lee (1984) for "astronomical silicates" and Mie theory (Bohren \& Huffman 


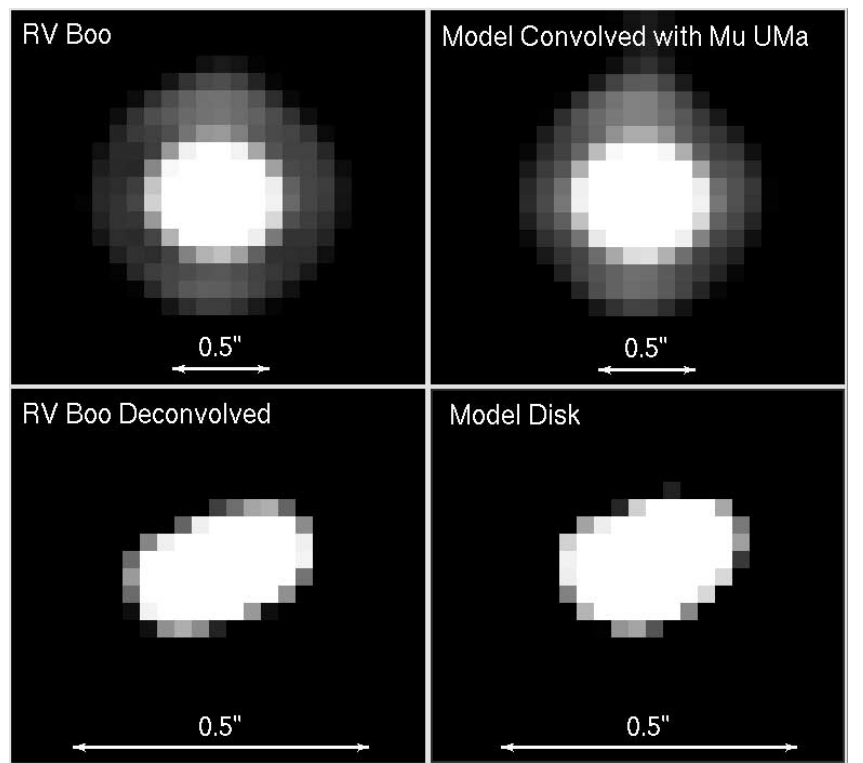

FIG. 8. - Comparison of RV Boo to the best-fit dust disk model $\left(r_{\text {inner }}=3 \mathrm{AU}\right.$, $r_{p}=40 \mathrm{AU}, \alpha \approx 3.3, \beta \approx 25$, total dust mass $m_{\text {dust }} \approx 3.17 \times 10^{27} \mathrm{~g} \approx 1.6 \times$ $10^{-6} M_{\odot}$, and an inclination from edge-on of $40^{\circ}$ ). For comparison with the raw RV Boo image, the model image in the bottom right has been convolved with the $\mu$ UMa PSF. North is up and east is to the left in all these images. Note that the RV Boo data is consistent with the best-fit SED model from Fig. 7. [See the electronic edition of the Journal for a color version of this figure.]

1983), we calculate the absorption cross sections of amorphous silicate grains as a function of size and their steady state temperatures (as a function of radial distance from the central star) in thermal equilibrium with the illuminating starlight intensity. We then obtain the dust model IR emission spectrum by integrating the dust emission over the entire size range and the entire disk and compare with the 12, 25, 60, and $100 \mu \mathrm{m}$ Infrared Astronomical Satellite (IRAS) broadband photometry, the 7.7$22.7 \mu \mathrm{m}$ low-resolution spectrum obtained with the IRAS Low Resolution Spectrometer (LRS; with a resolution of $\lambda / \Delta \lambda \approx$ $20)$, the 2.5-45 $\mu \mathrm{m}$ high-resolution spectrum obtained with the Short Wavelength Spectrometer (SWS) instrument (with a resolution of $\lambda / \Delta \lambda \approx 2000$ ) on board ISO, ${ }^{4}$ and the $9.8 \mu \mathrm{m}$ photometry presented in this work. We also compare the dust model image at $9.8 \mu \mathrm{m}$ convolved with the AO instrument PSF with the AO image of RV Boo and inclined at a variety of inclinations from edge-on. A number of models with $\beta \approx 25-30$ and inclinations from edge-on of $30^{\circ}-45^{\circ}$ fit the data to within $20 \%$ accuracy. In Figures 7 and 8, we respectively show the best-fit IR emission spectrum and disk image. The disk image is also shown convolved with the instrument PSF spectrum (see Fig. 7) and the AO image (see Fig. 8). The source properties (eccentricity, FWHM) for this model are plotted alongside source properties for the telescope data and PSF stars in Figure 5. This model, with $\alpha \approx 3.3$ and $\beta \approx 25$, has a total dust mass $m_{\text {dust }} \approx 3.17 \times 10^{27} \mathrm{~g} \approx 1.6 \times 10^{-6} M_{\odot}$ and an inclination from

\footnotetext{
4 The IRAS LRS spectrum integrated with the IRAS $12 \mu \mathrm{m}$ band filter function results in a factor of $\sim 9.8$ higher than the IRAS $12 \mu \mathrm{m}$ photometric flux. This is not surprising in view of the fact that RV Boo is a variable star. We have therefore reduced the flux level of the IRAS LRS spectrum by a factor of 9.8 to bring it into agreement with the IRAS $12 \mu \mathrm{m}$ broadband photometric data. Similarly, the flux level of the ISO SWS spectrum has also been reduced by a factor of $\sim 1.15$, in order to agree with the IRAS $12 \mu \mathrm{m}$ photometry.
}

edge-on of $40^{\circ}$. The dust spatial distribution peaks at $r_{p} \approx$ 40.5 AU. The maximum vertical visual optical depth is $\tau_{V} \approx$ 0.024 , confirming the validity of the optical-thin assumption made at the beginning of this modeling effort.

\section{DISCUSSION}

Where do the wide variety of PN shapes come from? Mass loss has practically ended by the proto-PN stage; therefore, any underlying structure that produces the shapes of PNs (via theories such as the GISW models) must form by the end of the AGB stage. While PNs possess an extremely wide range of morphologies, Meixner et al. (1999) found two primary mid-IR morphologies for proto-PNe corresponding to different values of optical depth: optically thick core/elliptical morphologies and optically thin toroidal morphologies. Ueta et al. (2000) found that each of these mid-IR morphologies also corresponds to a specific optical morphology. Optically thick core/elliptical mid-IR morphologies possess bipolar reflection nebulae and heavily obscured central stars (called DUPLEX by Ueta et al. 2000), while optically thin toroidal mid-IR morphologies possess elliptical reflection nebulae and nonobscured central stars (called SOLE by Ueta et al. 2000).

The proto-PNs observed by Meixner et al. (1999) are more evolved objects than AGB stars. Since mass loss has ceased by the proto-PN stage, the dense core/elliptical dust structures that produce DUPLEX sources (and are the precursors to structures formed during the PN stage) must have formed during the end of the AGB phases. AGB mass loss can be divided into two separate phases: an initial, spherically symmetric AGB wind $\left(\sim 10 \mathrm{~km} \mathrm{~s}^{-1}\right)$, supplanted by a faster superwind $\left(\sim 20 \mathrm{~km} \mathrm{~s}^{-1}\right)$ for a brief period at the end of the AGB phase (Ueta et al. 2000; Renzini 1981). With the onset of the superwind, mass-loss rates are expected to rise by factors of $\geq 10$ (Steffen et al. 1998). Ueta et al. (2000) propose that this superwind at the end of the AGB phases is intrinsically asymmetric, producing an equatorially flattened toroid that collimates later bipolar structure. However, it is not clear what mechanism could produce the asymmetry.

An AGB star like RV Boo that displays asymmetric structure is an excellent laboratory for studying the very beginnings of $\mathrm{PN}$ and proto-PN structure formation. RV Boo is one member of a set of AGB stars that display hallmarks of asymmetric structure. This small group of AGB stars $(\sim 20 \%)$ display very narrow CO line widths (as narrow as $\sim 1 \mathrm{~km} \mathrm{~s}^{-1}$, see Kahane et al. [1998], but generally $\leq 5 \mathrm{~km} \mathrm{~s}^{-1}$ ), with or without a broader underlying pedestal feature (with widths of 10-20 $\mathrm{km} \mathrm{s}^{-1}$ ). BM Gem displays just a narrow peak, while X Her (Kahane \& Jura 1996), RV Boo (Bergman et al. 2000), EP Aqr, RS Cnc, and IRC +50049 display narrow peaks as well as an underlying broader component. Jura \& Kahane (1999) interpret the narrow features in these objects as reservoirs of dust and molecular gas that are nearly at rest with respect to the central AGB stars. The broader CO line components are interpreted as spherical outflows or in some cases (RS Cnc and X Her) as bipolar outflows (Kahane \& Jura 1996).

RV Boo is unusual among these stars, since images of its $\mathrm{CO}$ emission suggest the presence of a large disk in Keplerian rotation (Bergman et al. 2000). One possible explanation for the presence of the disk is that mass loss from the AGB star has become equatorially enhanced through entrainment by a binary companion (Jura \& Kahane 1999; Morris 1987; Mastrodemos \& Morris 1998, 1999). Could this be the mechanism (in progress) that produces the dense collimating toroid of material invoked 
by Ueta et al. (2000), essentially, Ueta's equatorial superwind? While RV Boo does not have a known companion, a close companion ( $>100 \mathrm{AU})$ may be currently undetectable.

Interestingly, similar narrow $\mathrm{CO}$ line structure has been observed around a number of evolved post-AGB systems: two RV Tauri stars, AC Her (which has only a narrow line without a broad pedestal, suggesting perhaps that mass loss has ceased and only a molecular reservoir remains) and IRAS 08544-4431 (Maas et al. 2003), and the proto-PN Red Rectangle (Jura et al. 1995). In addition, a dusty disk has been detected around IRAS 085444431, and a Keplerian disk has been detected in $\mathrm{CO}$ around the Red Rectangle proto-PN (Bujarrabal et al. 2003). Are the disks around IRAS 08544-4431 and the Red Rectangle similar to that around RV Boo but at the corresponding later stages of evolution? AC Her (Van Winckel et al. 1998), IRAS 085444431, and the Red Rectangle all have known companions, a fact that supports theories of binary entrainment for disk formation.

If RV Boo is nearing the end of its AGB phase, the disk structure observed in CO and in the mid-IR may be the beginning of a denser disk/torus capable of collimating the fast winds produced during the PN phase. However, to determine whether the structure around RV Boo is the precursor of proto-PN and PN structure requires knowledge of the evolutionary status of RV Boo and also whether objects of a similar evolutionary status possess similar CO and mid-IR structure. RV Boo is a "red" $\mathrm{SRb}$, according to the classification system of Kerschbaum \& Hron (1992). They interpret the red SRb stars as a transitional stage between the blue SRb stars, which sit near the tip of the red giant branch, and Mira variables, which mark the tail end of the AGB phase. With a moderate mass-loss rate of $\sim 10^{-7} M_{\odot} \mathrm{yr}^{-1}$, RV Boo is probably not at the very tail end of AGB evolution. However, Kerschbaum \& Hron (1992) use single-star stellar models to constrain evolutionary state. If disk formation does require a binary companion, estimates of evolutionary state from such models may be inaccurate for RV Boo and similar objects.

While narrow CO lines have been found in $\sim 20 \%$ of AGB stars, it remains to be seen how common extended mid-IR structure such as that observed around RV Boo occurs in AGB stars. Indeed, even if mid-IR structure around narrow CO line AGB stars is common, it remains to be seen how similar RV Boo's CO and mid-IR structures are to those of other AGB stars with narrow $\mathrm{CO}$ lines. This is a comparison we cannot yet make, since no other similar object has both been mapped interferometrically in $\mathrm{CO}$ and imaged at high resolution in the mid-IR. Several of these objects share very similar observational properties with RV Boo, in particular, X Her, EP Aqr, RS Cnc, BM Gem, and EU And. It would be worthwhile to observe these objects both at high resolution in the mid-IR and also interferometrically in CO.

\section{CONCLUSIONS}

We present the first high-resolution $\left(0^{\prime \prime} 1\right)$, very high Strehl ratio $(0.97 \pm 0.03)$ mid-IR images of RV Boo utilizing the MMT adaptive secondary AO system. These are the first IR images presented in the literature with such high Strehl ratios; previous Strehl ratios for large telescopes have hardly exceeded $70 \%$ at any wavelength. RV Boo was observed at a number of wavelengths over two epochs $(9.8 \mu \mathrm{m}$ in 2003 May and 8.8, 9.8, and $11.7 \mu \mathrm{m}$ in 2004 February) and appeared slightly extended at all wavelengths. While the extension is within the measurement error for the 8.8 and $11.7 \mu \mathrm{m}$ data, the extension is more pronounced in the $9.8 \mu \mathrm{m}$ data. The slight extension seen in the $9.8 \mu \mathrm{m}$ data from both 2003 May and 2004 February suggests that the mid-IR structure around RV Boo is marginally resolved at $9.8 \mu \mathrm{m}$.

Because of our high Strehl ratios, which leads to extremely stable PSFs, we can deconvolve our images with those of PSF stars for a superresolution of $00^{\prime \prime} 1$. Based on the rotation of the extension with the sky and its non-PSF FWHM (at 8.8-11.7 $\mu \mathrm{m}$ ) and eccentricity, we conclude that the extension around RV Boo is indeed real and not an artifact of the telescope.

We tentatively detect a $\sim 60$ AU FWHM $(0.16$ at $390 \mathrm{pc})$ disk at $9.8 \mu \mathrm{m}$ around RV Boo. Previously, Bergman et al. (2000) found a $4^{\prime \prime}$ disk in $\mathrm{CO}$ with a position angle of $150^{\circ}$; we find a position angle of $120^{\circ} \pm 6^{\circ}$ for the $9.8 \mu \mathrm{m}$ disk. We measure a total disk flux of $145 \pm 24 \mathrm{Jy}$ at $9.8 \mu \mathrm{m}$. We closely reproduce the observed IR spectral energy distribution and the AO image in terms of an optically thin dust disk consisting of amorphous silicates with a power-law size distribution. We estimate a disk inclination angle of $30^{\circ}-45^{\circ}$ from edge-on and a disk dust mass of $1.6 \times 10^{-6} M_{\odot}$.

These MMT observations were made possible by the hard work of the entire Center for Astronomical Adaptive Optics (CAAO) staff at the University of Arizona. In particular, we would like to thank Tom McMahon, Kim Chapman, Doris Tucker, and Sherry Weber for their endless support of this project. The widefield AO CCD was installed by graduate student Nick Siegler. Graduate student Wilson Liu helped run the MIRAC3 camera during the run. The adaptive secondary mirror is a joint project of University of Arizona and the Italian National Institute of Astrophysics-Arcetri Observatory. We would also like to thank the whole MMT staff for their excellent support and flexibility during our commissioning run at the telescope.

The secondary mirror development was supported by the Air Force Office of Scientific Research under grant AFOSR F49620-00-1-0294. B. A. B., L. M. C., and D. P. acknowledge support from NASA Origins grant NAG5-12086 and NSF SAA grant AST-0206351. J. H. B. acknowledges support from NSF grants AST-9987408 and AST-0307687.
Balick, B., \& Frank, A. 2002, ARA\&A, 40, 439

Bergman, P., Kerschbaum, F., \& Olofsson, H. 2000, A\&A, 353, 257

Bohren, C. F., \& Huffman, D. R. 1983, Absorption and Scattering of Light by Small Particles (New York: Wiley)

Brusa, G., et al. 2003, Proc. SPIE, 4839, 691

Bujarrabal, V., Neri, R., Alcolea, J., \& Kahane, C. 2003, A\&A, 409, 573

Close, L. M., et al. 2003, ApJ, 598, L35

Draine, B. T., \& Lee, H. M. 1984, ApJ, 285, 89

Draine, B. T., \& Li, A. 2001, ApJ, 551, 807

Hinz, P. M., et al. 2000, Proc. SPIE, 4006, 349

Hoffman, W., et al. 1998, Proc. SPIE, 3354, 647

Hofmann, K.-H., Balega, Y., Blocker, T., \& Weigelt, G. 2001, A\&A, 379, 529

Jeffers, H. M., \& Vasilevskis, S. 1978, AJ, 83, 411

\section{REFERENCES}

Jura, M., Balm, S. P., \& Kahane, C. 1995, ApJ, 453, 721

Jura, M., \& Kahane, C. 1999, ApJ, 521, 302

Kahane, C., Barnbaum, C., Uchida, K., Balm, S. P., \& Jura, M. 1998, ApJ, 500,466

Kahane, C., \& Jura, M. 1996, A\&A, 310, 952

Kerschbaum, F., \& Hron, J. 1992, A\&A, 263, 97

Kerschbaum, F., \& Olofsson, H. 1999, A\&AS, 138, 299

Knapp, G. R., Young, K., Lee, E., \& Jorissen, A. 1998, ApJS, 117, 209

Kurucz, R. L. 1979, ApJS, 40, 1

Maas, T., Van Winckel, H., Lloyd Evans, T., Nyman, L.-A., Kilkenny, D., Martinez, P., Marang, F., \& van Wyk, F. 2003, A\&A, 405, 271

Mason, B. D., Wycoff, G. L., Hartkopf, W. I., Douglass, G. G., \& Worley, C. E. 2001, AJ, 122, 3466 
Mastrodemos, N., \& Morris, M. 1998, ApJ, 497, 303 1999, ApJ, 523, 357

Meixner, M., et al. 1999, ApJS, 122, 221

Morris, M. 1987, PASP, 99, 1115

Olofsson, H., Gonzalez Delgado, D., Kerschbaum, K., \& Shöier, F. L. 2002, A\&A, 391, 1053

Perryman, M. A. C., et al. 1997, A\&A, 323, L49

Renzini, A. 1981, in Physical Processes in Red Giants, ed. I. Iben \& A. Renzini (Dordrecht: Reidel), 431

Roddier, F., Roddier, C., Graves, J. E., \& Northcott, M. J. 1995, ApJ, 443, 249
Steffen, M., Szczerba, R., \& Schönberner, D. 1998, A\&A, 337, 149

Thorstensen, J. R. 2001, Skycalc User's Manual (Tucson: NOAO)

Ueta, T., Meixner, M., \& Bobrowsky, M. 2000, ApJ, 528, 861

Van Winckel, H., Waelkens, C., Waters, L. B. F. M., Molster, F. J., Udry, S., \& Bakker, E. J. 1998, A\&A, 336, L17

Vinkovic, D., Blocker, T., Hofmann, K.-H., Elitzur, M., \& Weigelt, G. 2004, MNRAS, 352, 852

Wildi, F., Brusa, G., Riccardi, A., Lloyd-Hart, M., Martin, H. M., \& Close, L. M. 2003, Proc. SPIE, 4839, 155 